Radioprotection, DunoD, 1975

Vol. $10, \mathrm{n}^{\circ} 4$, pages $181-203$

\title{
Vol à haute altitude et rayonnements cosmiques
}

\author{
I. CARACTÉRISTIQUES PHYSIQUES \\ DES TRANCHES D'ALTITUDE INFÉRIEURES A $28 \mathrm{~km}$
}

\author{
R.-P. DELAHAYE (*)
}

(Manuscrit reşu le 17 septembre 1975)

\begin{abstract}
RÉSUMÉ
La première partie de ce travail étudie les caractéristiques physiques de la tranche d'altitude comprise entre 0 et $28 \mathrm{~km}$. Le rayonnement cosmique galactique entraîne des irradiations pour les vols au-dessus de $15 \mathrm{~km}$ dont l'équivalent de dose varie de 0,5 à $2 \mathrm{mrem} / \mathrm{h}$, les valeurs les plus élevées étant rencontrées dans les vols polaires. Le rayonnement cosmique solaire apparaît surtout au cours des éruptions solaires dont l'influence peut se faire ressentir dans les tranches d'altitude supérieures à $15 \mathrm{~km}$. Cette répartition des événements solaires à protons montre que cette éventualité, si elle est rare, ne doit pas être négligée.
\end{abstract}

\begin{abstract}
In this first part, the physical characteristics of the $0-28 \mathrm{~km}$ altitude interval are studied. In the case of flights above $15 \mathrm{~km}$, galactic cosmic radiations result in exposures with dose equivalent rates ranging from 0.5 to $2 \mathrm{mrem} / \mathrm{h}$, the highest values corresponding to polar flights. Solar cosmic radiations occur chiefly during solar flares whose effects can be felt at altitudes above $15 \mathrm{~km}$. The distribution of proton solar events indicates that this occurence, though scarce, should not be neglected.
\end{abstract}

Les avions commerciaux supersoniques (tels que le Concorde) volent à plus de $15 \mathrm{~km}$ d'altitude. Bientôt, les avions de chasse ou de reconnaissance atteindront couramment 25 à $30 \mathrm{~km}$.

Ces séjours plus ou moins longs en altitude entrainnent une irradiation à faible débit de dose. Depuis quarante années, de très nombreux travaux ont précisé le débit de dose du rayonnement cosmique et de ses composantes,

(*) Service de Radiologie, Hôpital Begin, 69, avenue de Paris, 94160 Saint-Mandé.

RADIOPROTECTION, VOL. $10-\mathrm{N}^{\circ} 4$ 
ses variations. Les recherches biologiques, malgré leur multitude, n'ont pas, à ce jour, déterminé toutes les particularités de l'irradiation par les rayonnements cosmiques.

Nous étudierons successivement :

- les caractéristiques physiques des tranches d'altitude inférieures à $28 \mathrm{~km}$ (première partie);

- les recherches biologiques entreprises et la discussion des faits observés (deuxième partie).

\section{CARACTÉRISTIQUES PHYSIQUES DE LA TRANCHE D'ALTITUDE $0-28 \mathrm{~km}$}

Dès que l'altitude augmente, l'irradiation du personnel navigant est due à deux variétés du rayonnement cosmique :

- le rayonnement cosmique galactique (RCG) avec ses deux principales composantes ionisante et neutronique;

- le rayonnement cosmique solaire émis au cours de certaines éruptions. tique.

La majeure partie de la dose provient du rayonnement cosmique galac-

\subsection{Les doses dues aU RayonNement cosmiQue galactiQue}

Au-delà de $40 \mathrm{~km}$ d'altitude, le rayonnement cosmique galactique s'observe à l'état pur. Il est constitué de noyaux atomiques complètement ionisés et fortement accélérés. C'est le rayonnement cosmique galactique primaire.

Au fur et à mesure de la pénétration dans l'atmosphère terrestre, ces noyaux sont désintégrés au cours de réactions nucléaires avec l'azote et l'oxygène de l'air. Ainsi se crée le rayonnement cosmique galactique secondaire qui comprend des neutrons, des mésons, des électrons, des positons, etc.

\subsubsection{Le rayonnement cosmique galactique primaire}

Il est formé par : des protons (75 à 80 p. cent), des particules alpha (15 à 19 p. cent), des ions lourds $(Z>2)(1$ à 2 p. cent), très peu de rayonnement gamma $\left(3 \pm 1\right.$ photon $/ \mathrm{cm}^{2}$.s.sr).

Le terme ion lourd s'applique aux particules du rayonnement cosmique galactique primaire et secondaire de charge supérieure à 2. Ces particules sont groupées en cinq catégories (tableau I).

Les particules du rayonnement cosmique galactique primaire se déplacent à des vitesses voisines de celles de la lumière. Elles peuvent atteindre des énergies de $10^{18}$ à $10^{20} \mathrm{eV}$. 
TABLEAU I

\begin{tabular}{|l|c|c|}
\hline \multicolumn{1}{|c|}{ GROUPE } & $\mathrm{Z}$ & NOYAU ATOMIQUE \\
\hline $\mathrm{L}:$ Light (léger) & $3-5$ & $\mathrm{Li}, \mathrm{Be}, \mathrm{B}$ \\
\hline $\mathrm{M}:$ Medium (moyen) & $6-9$ & $\mathrm{C}, \mathrm{N}, \mathrm{O}, \mathrm{F}$ \\
\hline $\mathrm{H}:$ Heavy (lourd) & $10-19$ & $\mathrm{Ne}$ à $\mathrm{K}$ \\
\hline V.H : Very heavy (très lourd) & $20-28$ & $\mathrm{Ca}$ à Ni \\
\hline $\begin{array}{l}\text { S.H : Super Heavy (extrêmement } \\
\text { lourd) }\end{array}$ & $>28$ & \\
\hline
\end{tabular}

\subsubsection{Le rayonnement cosmique galactique secondaire}

Par des réactions nucléaires complexes, les particules du rayonnement cosmique galactique primaire créent des produits secondaires par le phénomène de l'étoile. Les branches de cette étoile sont la matérialisation de ces produits secondaires qui peuvent, eux-mêmes, posséder une énergie suffisante pour créer une nouvelle interaction nucléaire. Le mécanisme se développe en cascade jusqu'au moment où les produits de réaction ne possèdent plus d'énergie suffisante pour provoquer de nouvelles interactions.

Il existe deux composantes :

- une dure ou pénétrante formée de nucléons (protons, neutrons) et de mésons $\mu$;

- une molle (mésons $\pi$ neutres, électrons, etc.).

\subsubsection{Débit de dose du RCG}

Les mesures effectuées ont porté sur la composante ionisante et sur la composante neutronique.

\subsubsection{Mesures effectuées sur la composante ionisante}

La variation la plus importante est due à l'altitude dont les effets sont déterminés par la quantité de matière que les radiations ont traversée, plutôt que par l'altitude elle-même. La figure 1 précise la relation entre l'altitude et la masse sus-jacente par unité de surface pour une atmosphère standard.

Trois niveaux de vol ont été plus particulièrement étudiés [2] :

- 10000 à $13000 \mathrm{~m}\left(200 \mathrm{~g} / \mathrm{cm}^{2}\right)$ pour les avions subsoniques; à Mach 2;

-16000 à $18000 \mathrm{~m}\left(100 \mathrm{~g} / \mathrm{cm}^{2}\right)$ pour les avions supersoniques volant

- $28000 \mathrm{~m}\left(20 \mathrm{~g} / \mathrm{cm}^{2}\right)$ pour les avions volant à Mach 3.

voL. $10-\mathrm{N}^{\circ} 4$ 


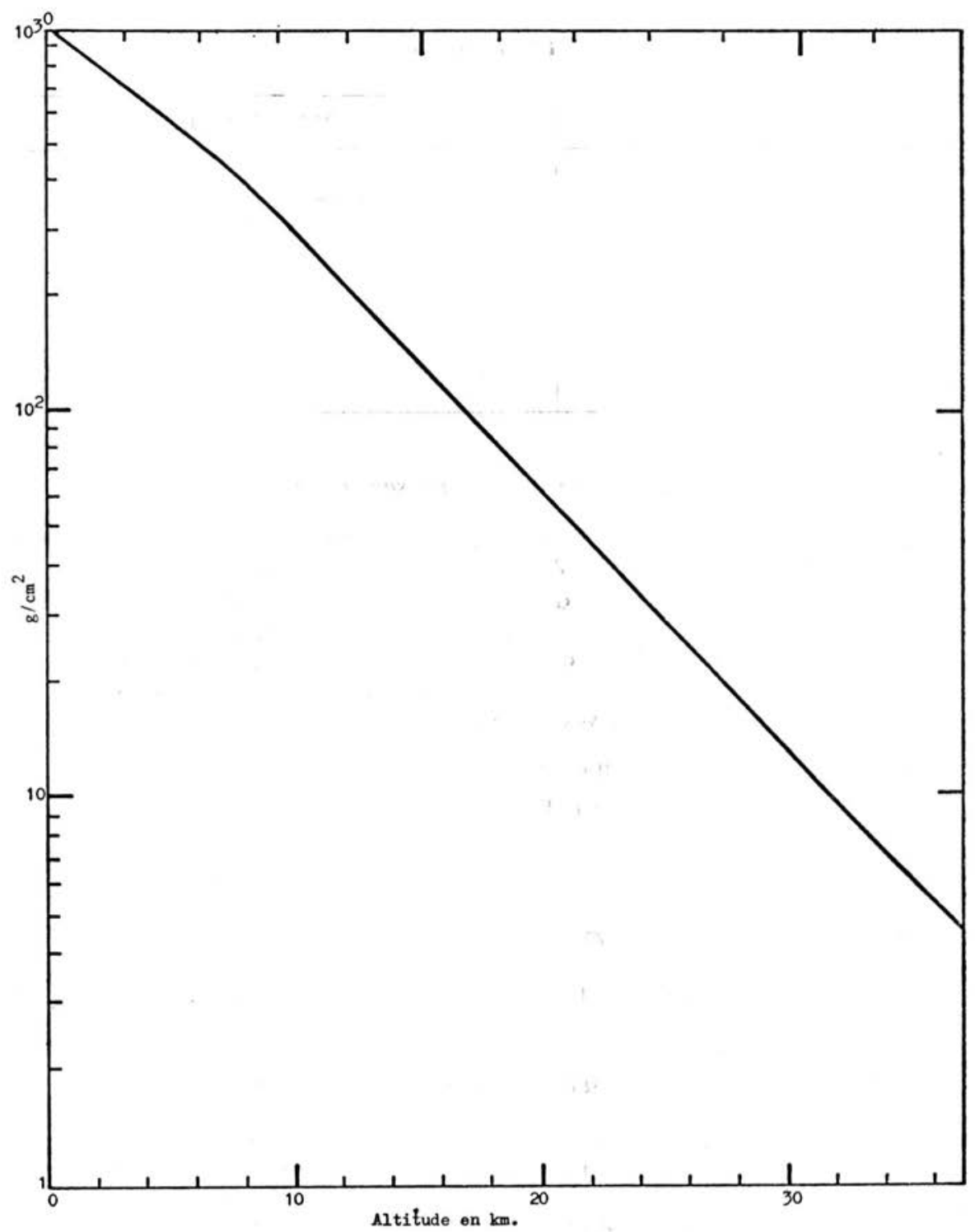

FIG. 1. - Rapport entre l'altitude et l'atmosphère résiduelle $\left(\mathrm{g} / \mathrm{cm}^{2}\right)$.

La plus grande partie des mesures de l'ionisation à des altitudes allant jusqu'à $20 \mathrm{~g} / \mathrm{cm}^{2}(28000 \mathrm{~m})$ est réalisée avec des chambres d'ionisation à remplissage d'argon et étalonnées au sol, en utilisant des champs connus de rayonnements gamma. Ceux-ci peuvent être convertis en débit de dose 


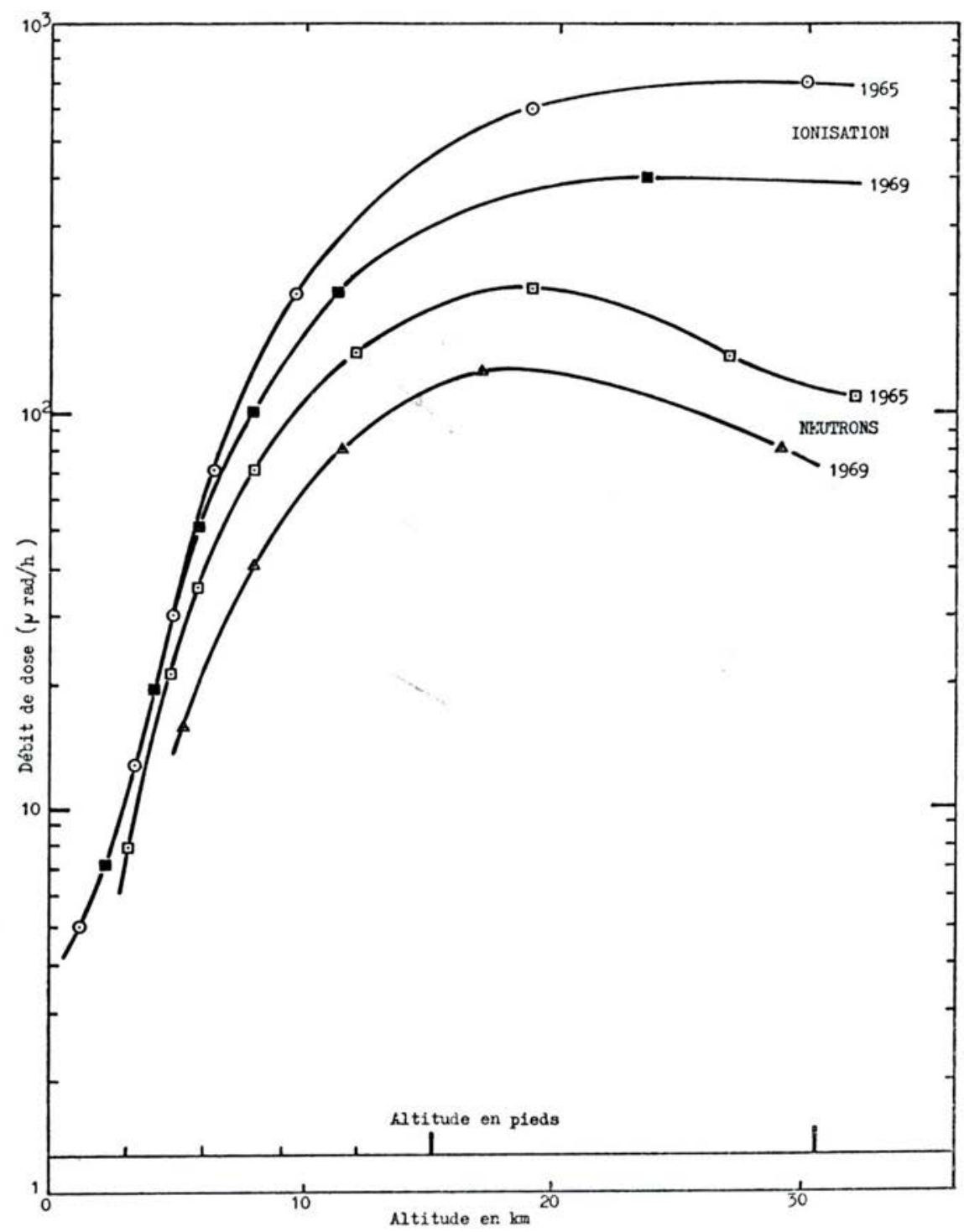

FIG. 2. - Variation du débit de dose avec l'altitude (latitudes élevées).

absorbée en supposant que les rapports entre les forces d'arrêt et d'énergie nécessaires à la création d'une paire d'ions dans l'argon et dans l'air sont les mêmes pour la composante ionisante du rayonnement cosmique et pour le rayonnement gamma [22].

vol. $10-\mathrm{N}^{\circ} 4$ 


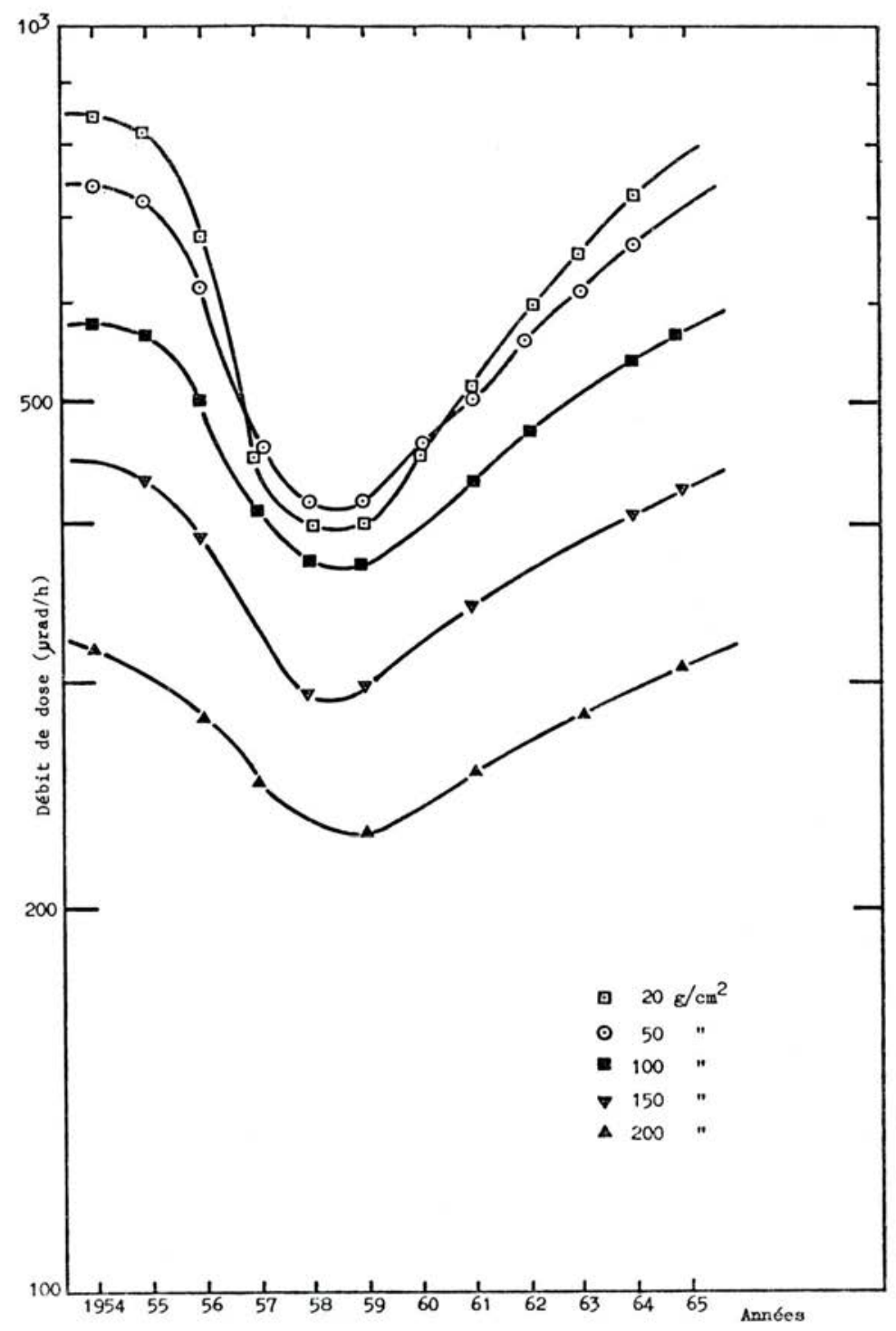

Fig. 3. - Variation du débit de dose aux latitudes élevées.

La figure 2 précise la variation du débit de dose avec l'altitude, à une latitude élevée, pour les deux composantes ionisante et neutronique.

La figure 3 montre la variation du débit de dose pendant un cycle solaire, à latitude élevée, pour des altitudes choisies exprimées en grammes par centimètre carré. 


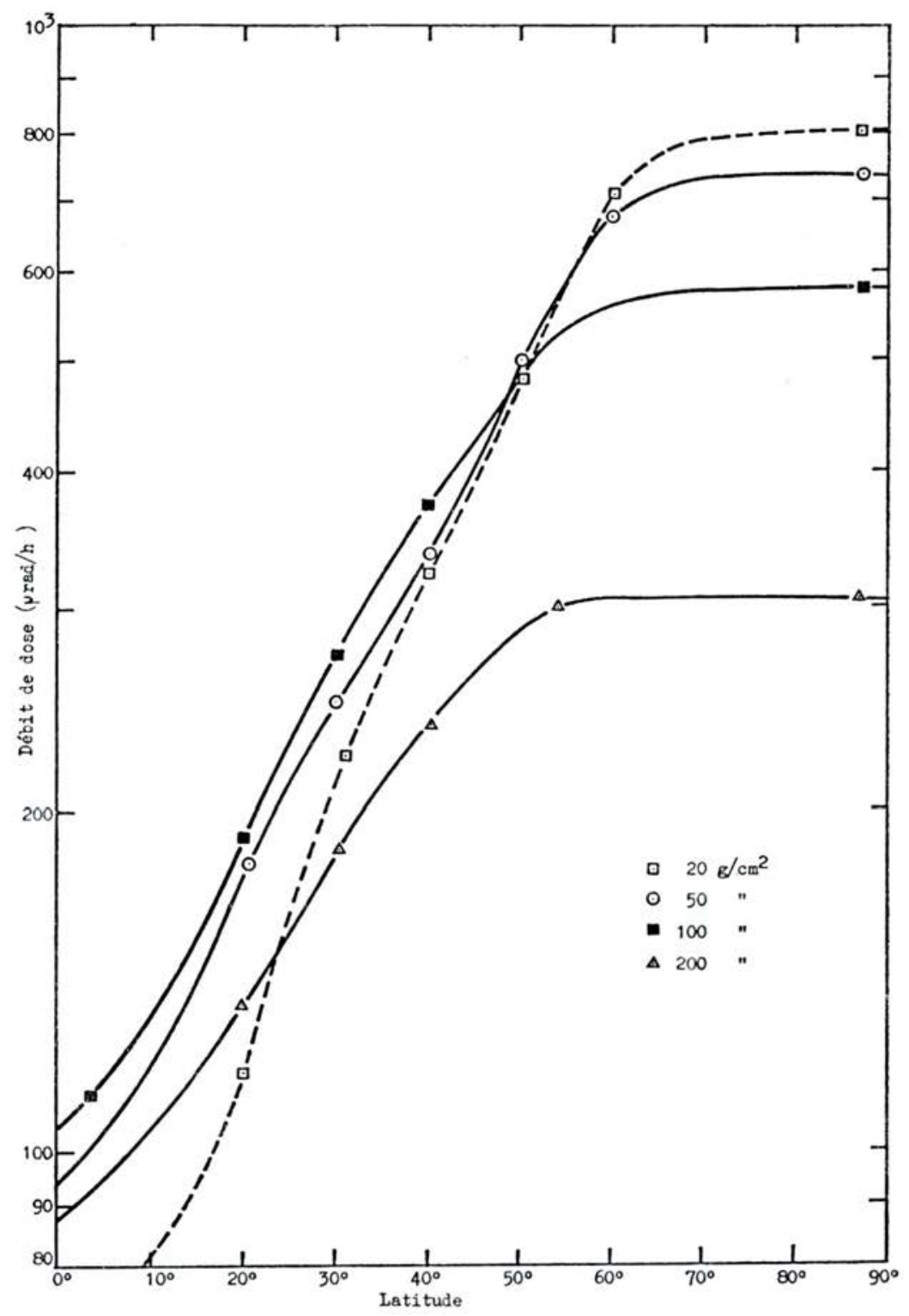

Fig. 4. - Variation du débit de dose en fonction de la latitude (activité solaire minimale).

Les figures 4 et 5 démontrent la variation du débit de dose avec la latitude pour des altitudes choisies (périodes de minimum et de maximum d'activité solaire). E. W. FULLER fait remarquer que ces courbes s'appliquent aux 


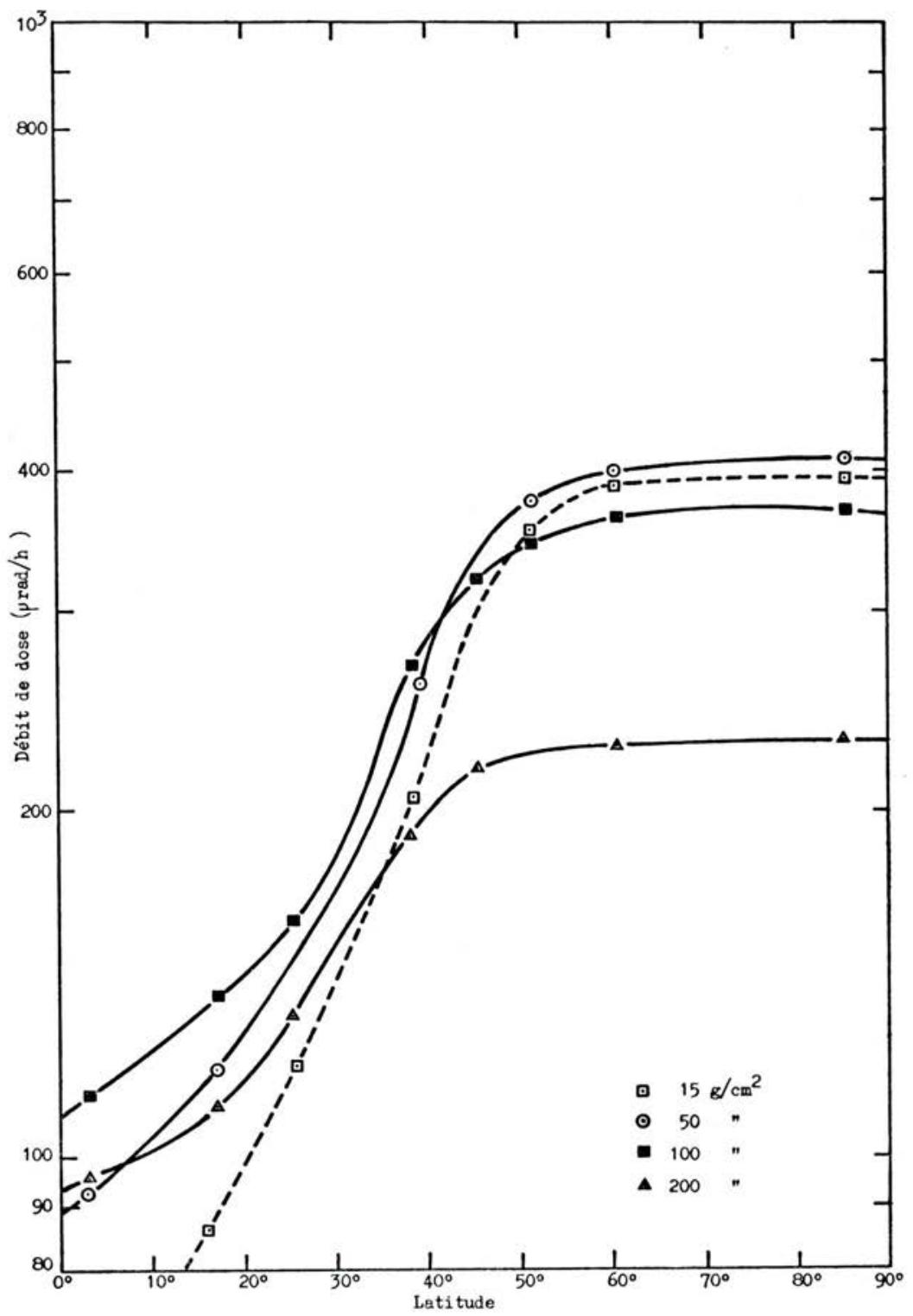

Fig. 5. - Variation du débit de dose en fonction de la latitude (activité solaire maximale).

doses dans de petits volumes isolés de tissu, en pratique, aux doses au niveau des extrémités. Comme cette composante de la dose varie lentement avec la 
profondeur, sauf pour les altitudes élevées, elle est aussi une mesure raisonnable de la dose à travers le corps [22].

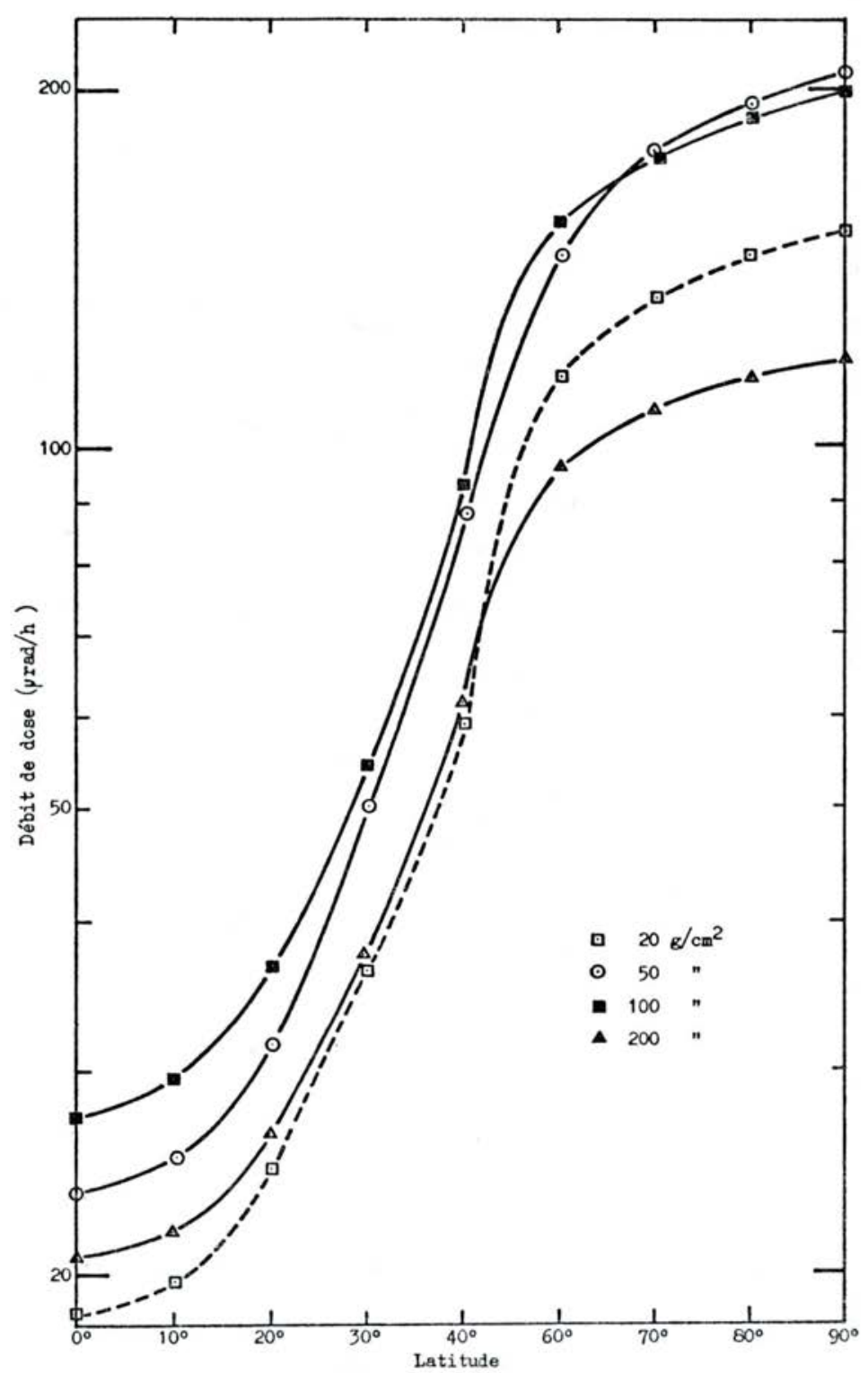

FIG. 6. - Débit de la dose due aux neutrons en fonction de la latitude (activité solaire minimale). 


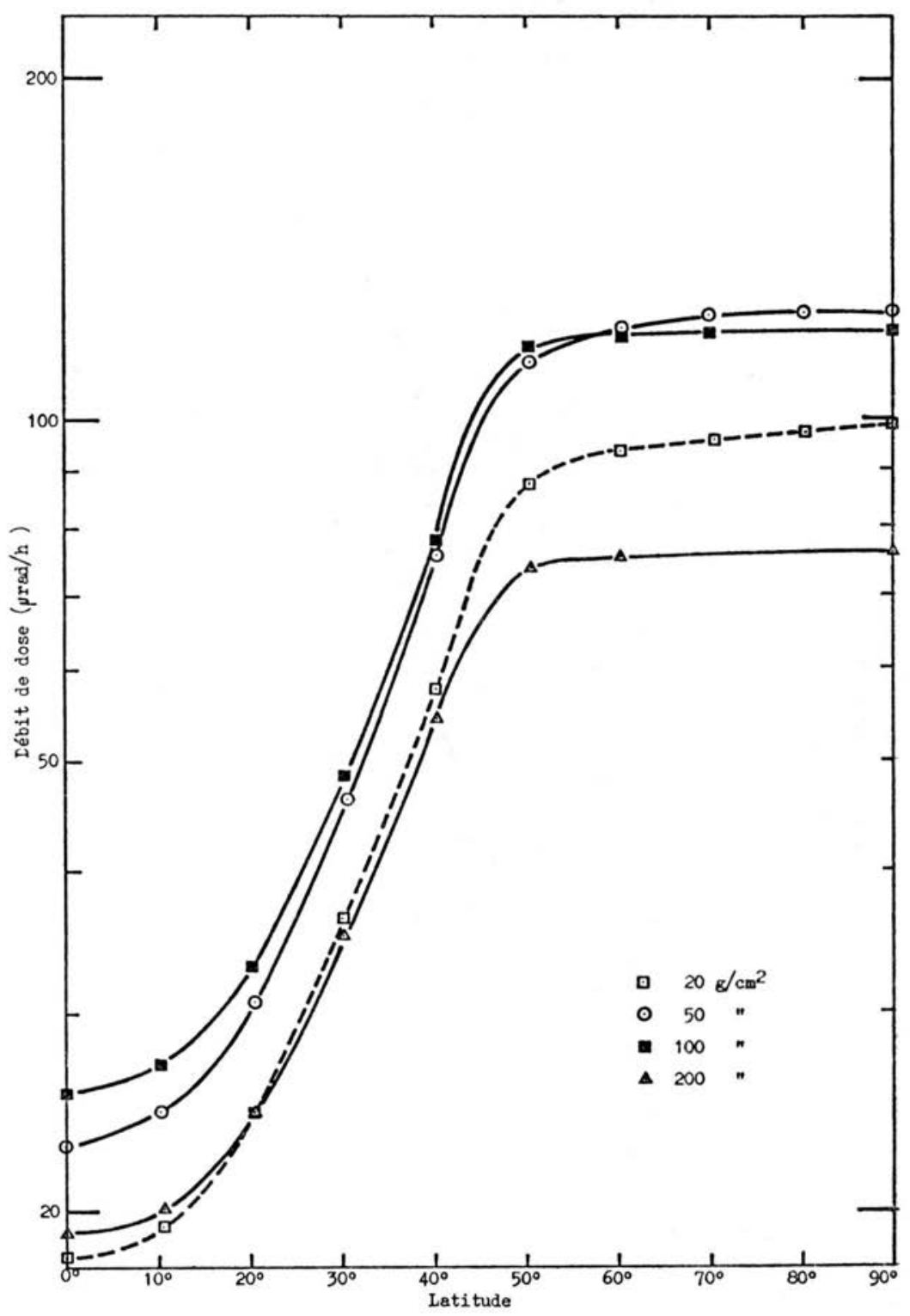

Fig. 7. - Variation du débit de dose due aux neutrons en fonction de la latitude (activité solaire maximale). 


\section{L'examen des différentes courbes montre que :}

$1^{\circ}$ dans le domaine des altitudes étudiées, l'intensité de dose varie peu entre 60 et $90^{\circ}$ de latitude géomagnétique;

$2^{\circ}$ que le débit de dose à l'équateur géomagnétique varie entre un facteur 6 (altitude élevée, minimum solaire) et 2,5 (basse altitude) en fonction de l'altitude et de la phase du cycle solaire;

$3^{\circ}$ que la variation pendant le cycle solaire est plus grande aux altitudes et aux latitudes élevées (près du pôle); il y a une variation d'un facteur 1,8.

\subsubsection{Mesure de la composante neutronique}

Il apparaît, au cours des interactions des particules du RCG primaire avec les composantes de l'atmosphère, des neutrons dont l'énergie se situe dans deux domaines : l'un d'énergie très élevée, l'autre de faible énergie (inférieure à $1 \mathrm{MeV}$ ).

Près de la moitié de la dose vient des neutrons d'énergie élevée. On observe des variations de la dose dues aux neutrons avec le temps, l'altitude et la latitude (fig. 6 et 7). E. W. Fuller (22] note que, dans le cas de la composante neutronique, la différence entre irradiation de petit et de grand volume est plus importante que pour la composante ionisante du RCG. Dans l'établissement des courbes des figures 2,6 et 7 , on considère que la dose la plus élevée résulte du fait qu'elle se rapporte à un petit volume.

\subsubsection{Mesure de la dose due aux étoiles nucléaires}

Les collisions qui produisent les neutrons créent des particules chargées qui, le long de leur trajet, possèdent une densité d'ionisation élevée.

Comme plusieurs particules chargées prennent naissance en un point, elles produisent une densité élevée d'énergie en ce point. En utilisant des émulsions nucléaires particulières (charge de l'émulsion par du glycérol ou modification de la teneur en argent), Fuller et Clarke [20] d'une part, et DAVIDSON [15] d'autre part, déterminent statistiquement le nombre d'étoiles. Les figures 8 et 9 (d'après SHEN [35]) montrent que la fréquence des étoiles varie en fonction de la latitude. Fuller estime que le débit de dose par étoile est environ 5 p. cent de celle de la dose par ionisation.

\subsubsection{Les ions lourds, d'après E. W. FULLER [22]}

1 à 2 p. cent des particules du RCG primaire ont un $Z$ compris entre 2 et 28 . La plupart font partie du groupe $\mathbf{M}$ des ions lourds. Le nombre des ions lourds décroît rapidement quand les radiations pénètrent dans l'atmosphère; néanmoins, ils sont encore détectables à des altitudes supérieures à $16500 \mathrm{~m}$. Le trajet des ions lourds présente deux caractéristiques :

$1^{\circ}$ Le taux minimal de perte d'énergie est proportionnel à $Z^{2}$, de sorte que, sur de longues portions de leur trajet, le TLE de ces ions lourds est plus élevé que celui atteint par les protons à la fin de leur trajectoire, c'est-à-dire 


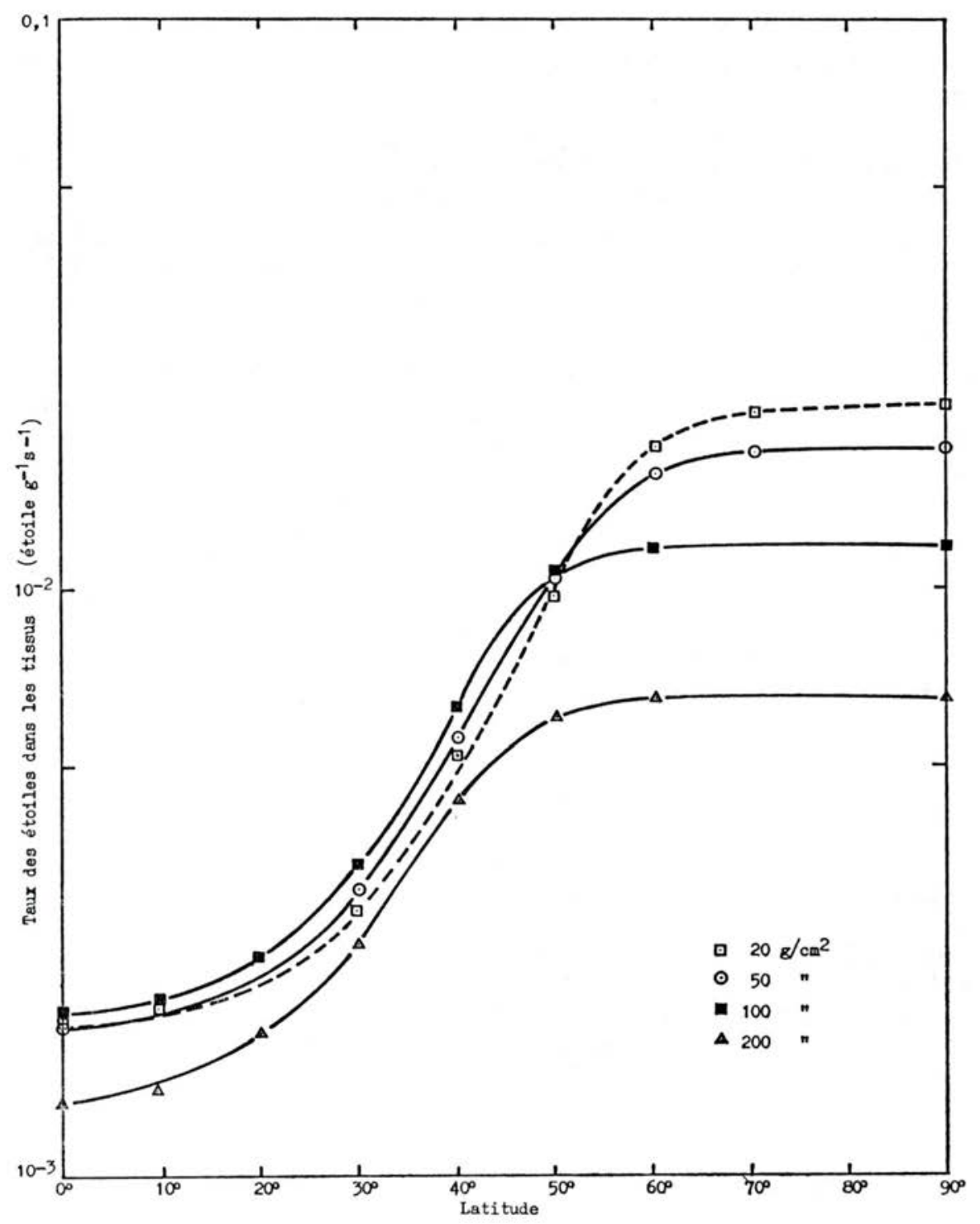

Fig. 8. - Variation de la fréquence des étoiles dans les tissus en fonction de la latitude (activité solaire minimale), d'après SHEN [35].

qu'un ion de néon d'énergie élevée $(Z=10)$ a un trajet de $1000 \mu \mathrm{m}$ où le TLE est supérieur à $100 \mathrm{keV} / \mu \mathrm{m}$; la longueur correspondante du trajet 'du proton est de $10 \mu \mathrm{m}$. 


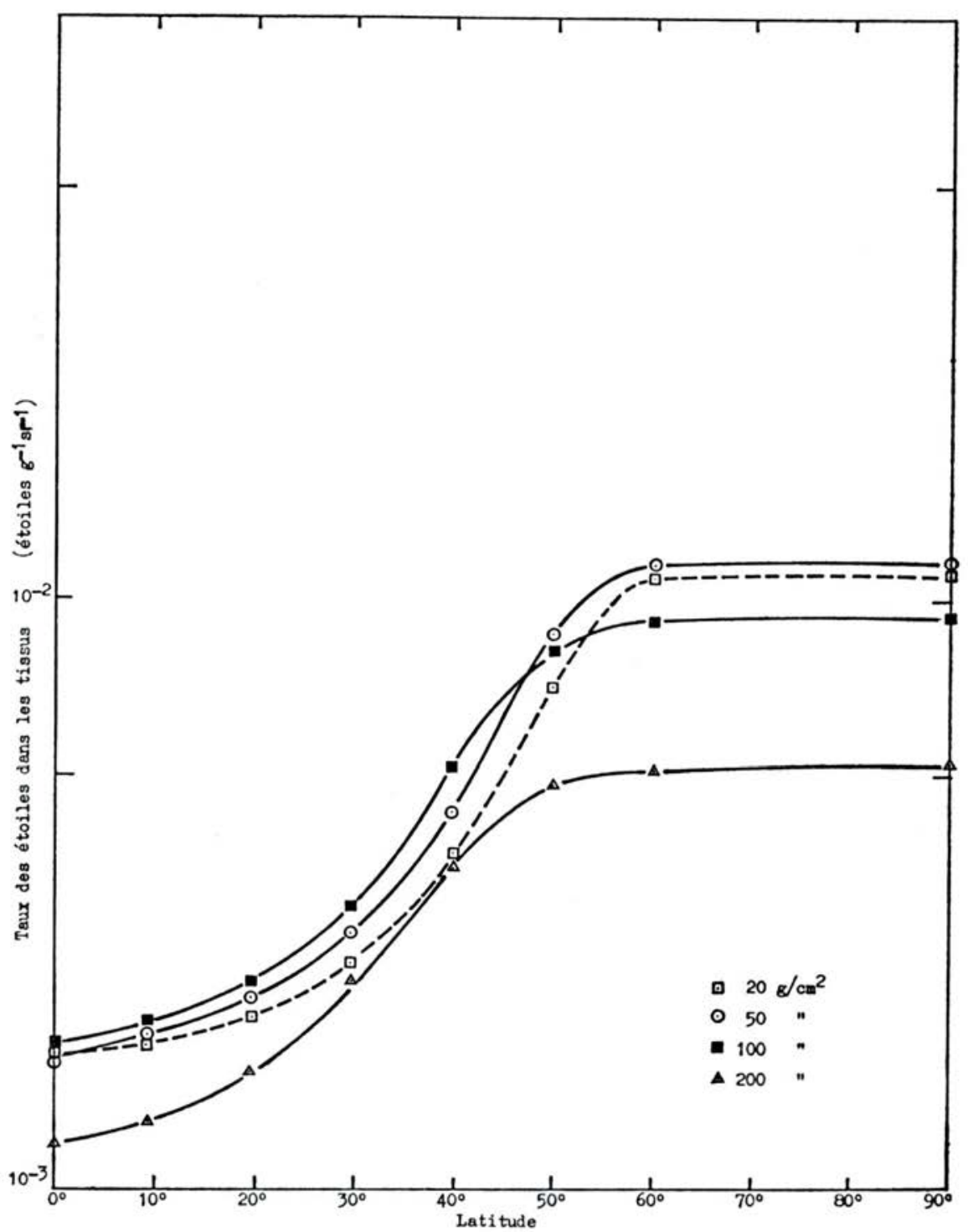

FIG. 9. - Variation de la fréquence des étoiles dans les tissus en fonction de la latitude (activité solaire maximale), d'après SHEN [35].

$2^{\circ}$ Le taux maximal de perte d'énergie, atteint près de la fin du trajet, varie aussi comme $Z^{2}$, et le TLE atteint ainsi des valeurs jamais obtenues par les particules plus légères : par exemple, le TLE pour un ion de néon dépasse $1500 \mathrm{keV} / \mu \mathrm{m}$ à $20 \mu \mathrm{m}$ de la fin de son trajet.

voL. $10-\mathrm{N}^{\circ} 4$ 


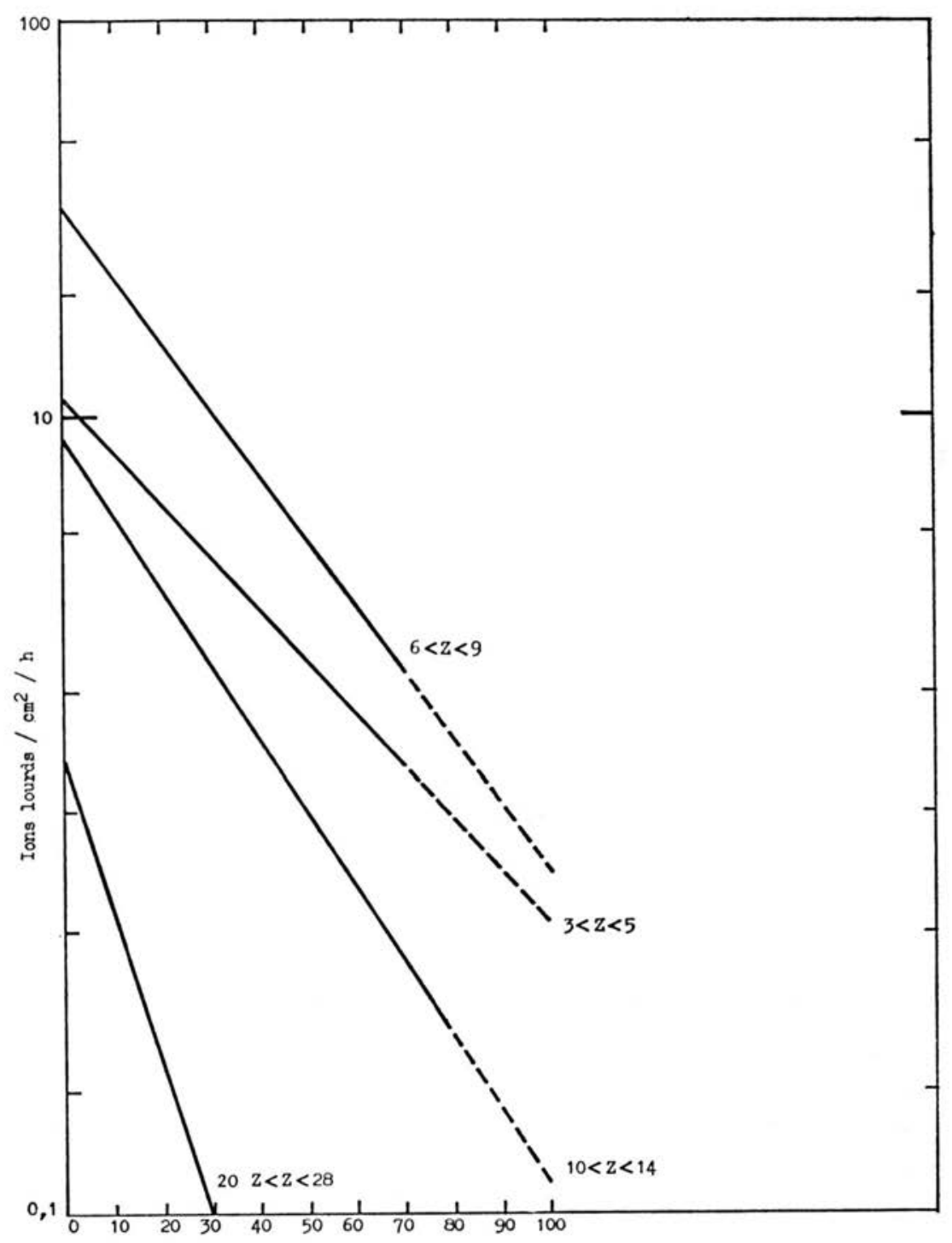

FIG. 10. - Variation du flux des ions lourds en fonction de la profondeur dans l'atmosphère, d'après ALLKofer [11].

Il est possible que ces deux caractéristiques puissent donner des effets non observés avec les radiations à TLE faible, de là vient l'intérêt spécial porté à ces particules. 


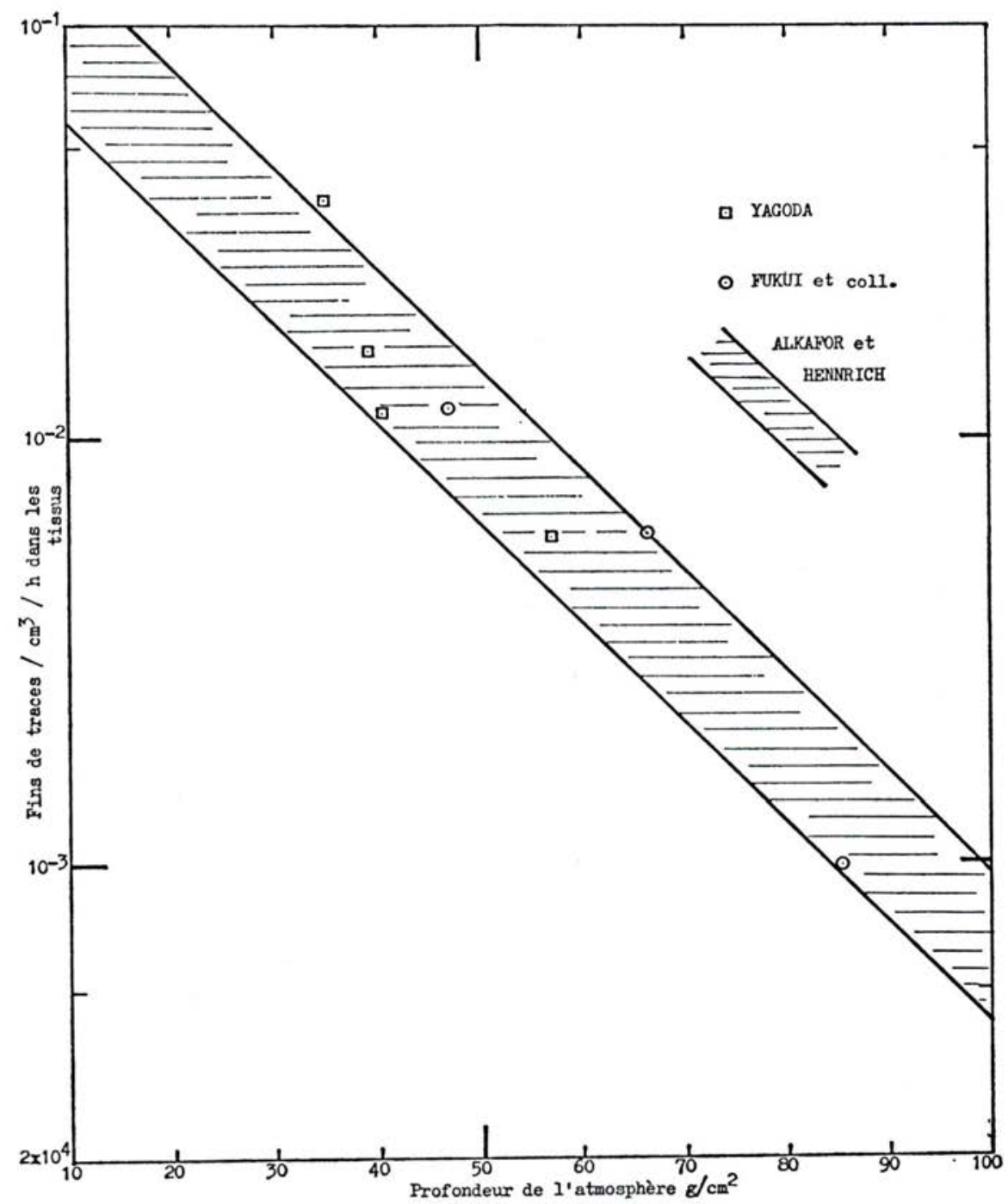

Fig. 11. - Fréquence des fins de traces des ions lourds dans les tissus, d'après ALLKOFER[11].

Les figures 10 et 11 , d'après un rapport d'AlLKOFER [1] montrent les résultats obtenus par son propre groupe et ceux de WEBBER. La figure 10 montre comment le flux d'ions lourds varie avec l'altitude, la figure 11 la densité des fins de traces, c'est-à-dire le nombre de régions très lourdement ionisées correspondant à la fin du trajet.

voL. $10-\mathrm{N}^{\circ} 4$ 


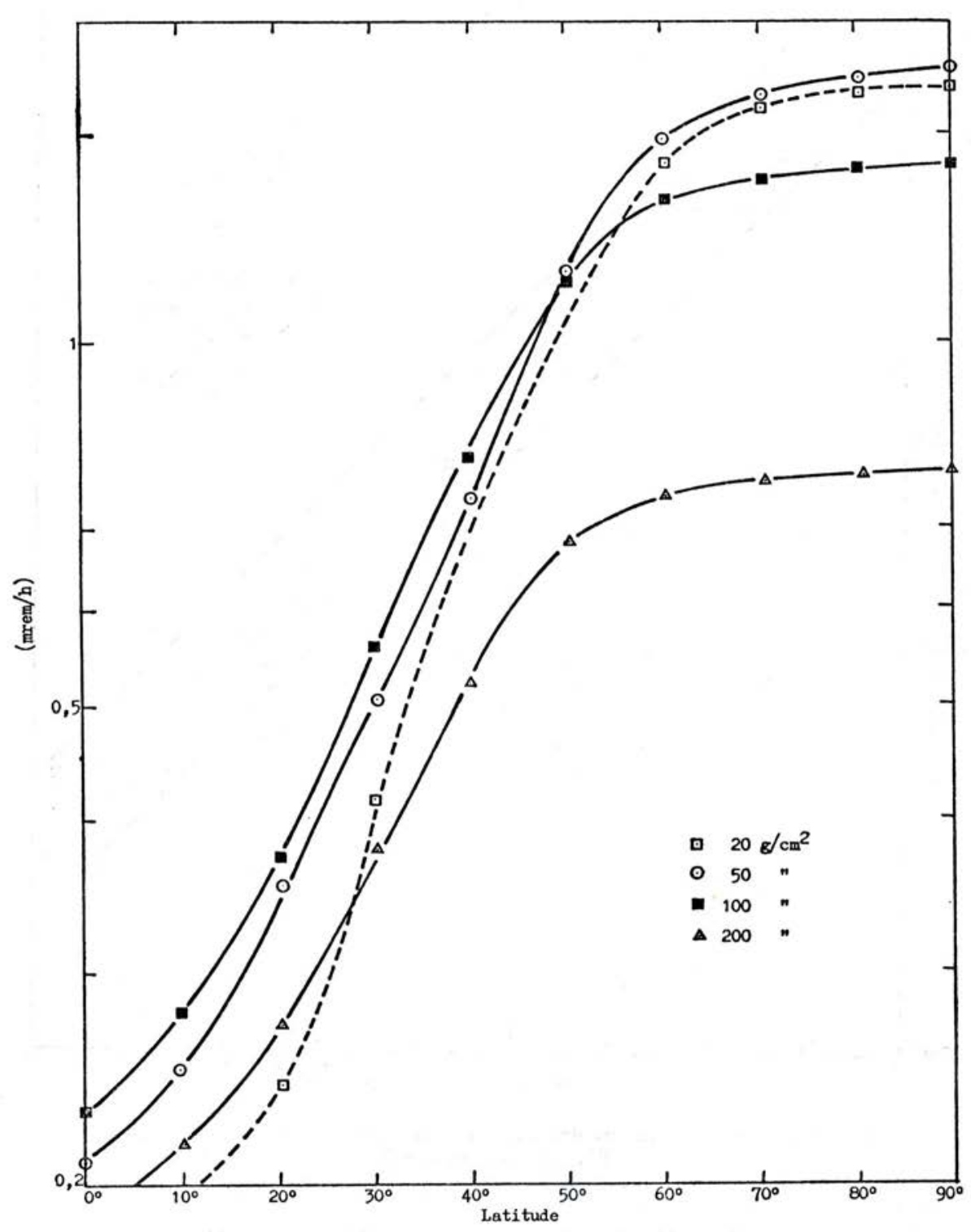

Fig. 12. - Variation du débit d'équivalent de dose avec la latitude (activité solaire minimale). 


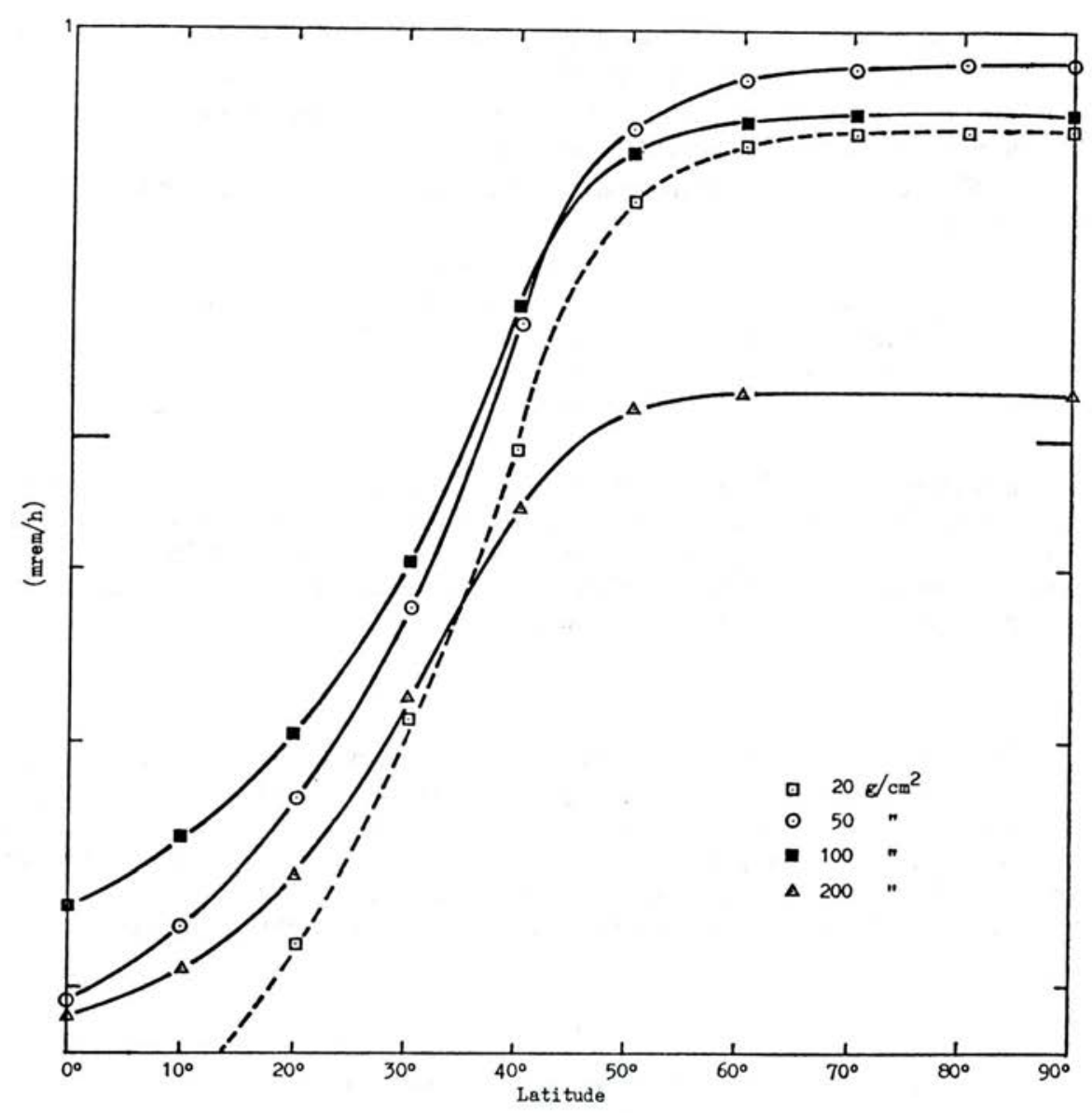

FIG. 13. - Variation du débit d'équivalent de dose avec la latitude (activité solaire maximale).

\subsubsection{5. Équivalents de dose due au RCG}

E. W. Fuller, confirmant les résultats de K. O'Brien et J. E. MacLaUGHLIN [29] remarque que la majeure partie des radiations primaires a un TLE faible $(\mathrm{FQ}=1)$, la composante à TLE élevé provenant de collisions nucléaires et des neutrons. Les mesures de H. J. SchaEfFer [34] montrent que le FQ de la partie de faible énergie (inférieure à $20 \mathrm{MeV}$ ) du spectre de neutrons est égale à 2,6 .

Les données expérimentales avec les étoiles nucléaires sont rares. Les mesures d'émulsion (Fuller et ClaRKe [22] et DAVIDSON [15]) donnent un équivalent de débit de dose de $0,4 \mathrm{mrem} / \mathrm{h}(0,05 \mathrm{mrad} / \mathrm{h}$ avec un $\mathrm{FQ}$ égal à 8 pour une altitude de $21000 \mathrm{~m}$ ).

VoL. $10-\mathrm{N}^{\circ} 4$ 
Fuller [22], Cowan et coll. [12] aboutissent à un FQ égal à 1,74 aux latitudes et altitudes élevées et au minimum d'activité solaire.

Les composantes à TLE élevé des étoiles nucléaires font passer effectivement le FQ de la composante ionisante à 1,5 .

E. W. Fuller [22] détermine le débit d'équivalent de dose par la relation suivante :

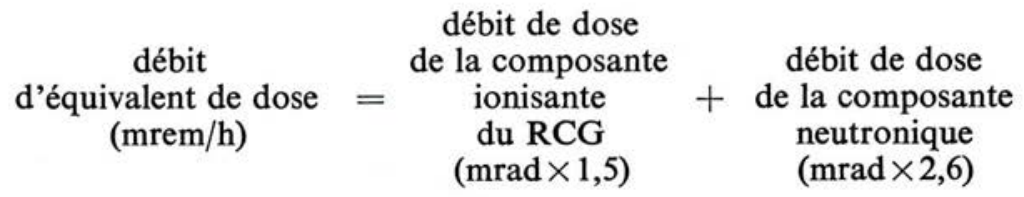

Les figures 12 et 13 donnent le débit d'équivalent de dose en millirem par heure. Ces valeurs ne comprennent pas la contribution des ions lourds et Fuller remarque que l'hypothèse suivante a été retenue : l'équivalent de dose provenant des étoiles nucléaires peut être calculé de la même façon que pour les trajets à TLE élevé isolés.

\subsection{LE RAYONNEMENT COSMIQUE SOLAIRE}

Le rayonnement cosmique solaire est émis par le soleil en association avec des éruptions solaires. La majeure partie des particules atteignant la Terre sont des protons, émis au cours de certaines éruptions (événements solaires à protons, ESP). La fréquence de ces derniers suit le cycle solaire de onze ans, mais, dans les années actives, ces événements se produisent au hasard. Ces ESP varient en durée, en quantité d'énergie et en nombre de protons émis.

\section{TABLEAU II}

NOMBRe D'ÉVÉNEMENTS SOLAIRES A PROTONS (ESP) AU $19^{\circ}$ CYCLE DÉPASSANT DES NIVEAUX CHOISIS DU DÉBIT D'ÉQUIVALENT DE DOSE, d'après Fuller et SimON [21].

\begin{tabular}{|l|l|l|l|l|l|l|l|l|l|l|l|}
\hline $\begin{array}{l}\text { Débit } \\
\text { d'équivalent de } \\
\text { dose mrem/h }\end{array}$ & 2000 & 1000 & 500 & 200 & 100 & 50 & 20 & 10 & 5 & 2 & 1 \\
\hline $\begin{array}{l}\text { Nombre d'ESP } \\
\text { dépassant à } 20 \mathrm{~g} / \mathrm{cm}^{2} \\
(28000 \mathrm{~m})\end{array}$ & $1 * *$ & 3 & 4 & 9 & 10 & 12 & 19 & 24 & 26 & 31 & 35 \\
\hline $\begin{array}{l}\text { Nombre d'ESP } \\
\text { dépassant à 100 } \mathrm{g} / \mathrm{cm}^{2} \\
(16-18000 \mathrm{~m})\end{array}$ & & $1 *$ & 1 & 2 & 4 & 9 & 13 & 17 & 24 \\
\hline
\end{tabular}

(*) 23 février $1956,500 \mathrm{mrem} / \mathrm{h}$.

(**) 23 février 1956, $2400 \mathrm{mrem} / \mathrm{h}$. 


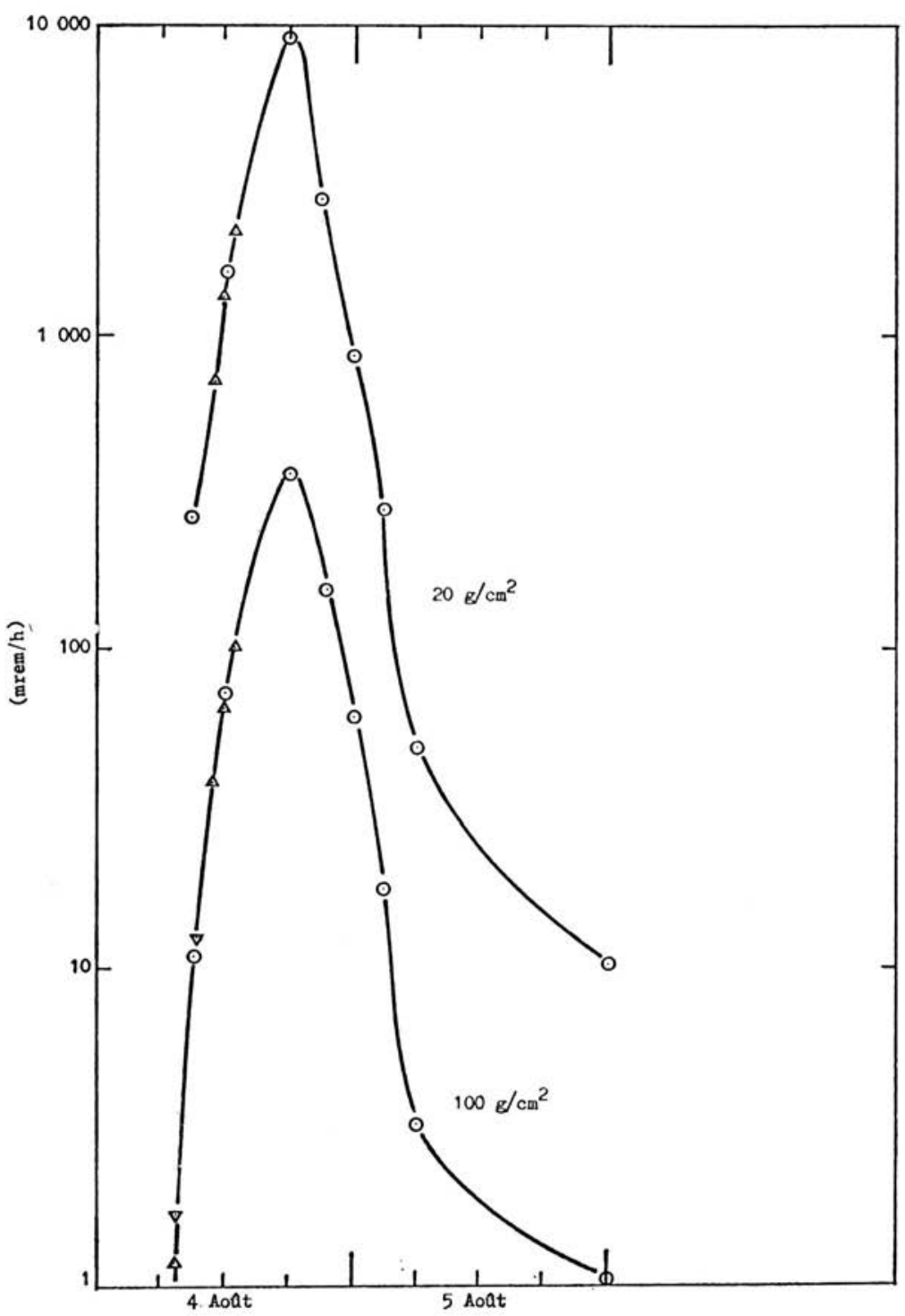

Fig. 14. - Variation du débit d'équivalent de dose avec le temps à 20 et $100 \mathrm{~g} / \mathrm{cm}^{2}$, événement solaire d'août 1972, d'après FulLER [14].

Quelques événements solaires à protons, notamment celui du 23 février 1956, ont entraîné des irradiations importantes dans l'espace.

Jusqu'ici, il a été très difficile d'enregistrer sur des avions ces événements irréguliers. La majeure partie de nos connaissances actuelles sur les doses

vol. $10-\mathrm{N}^{\circ} 4$ 


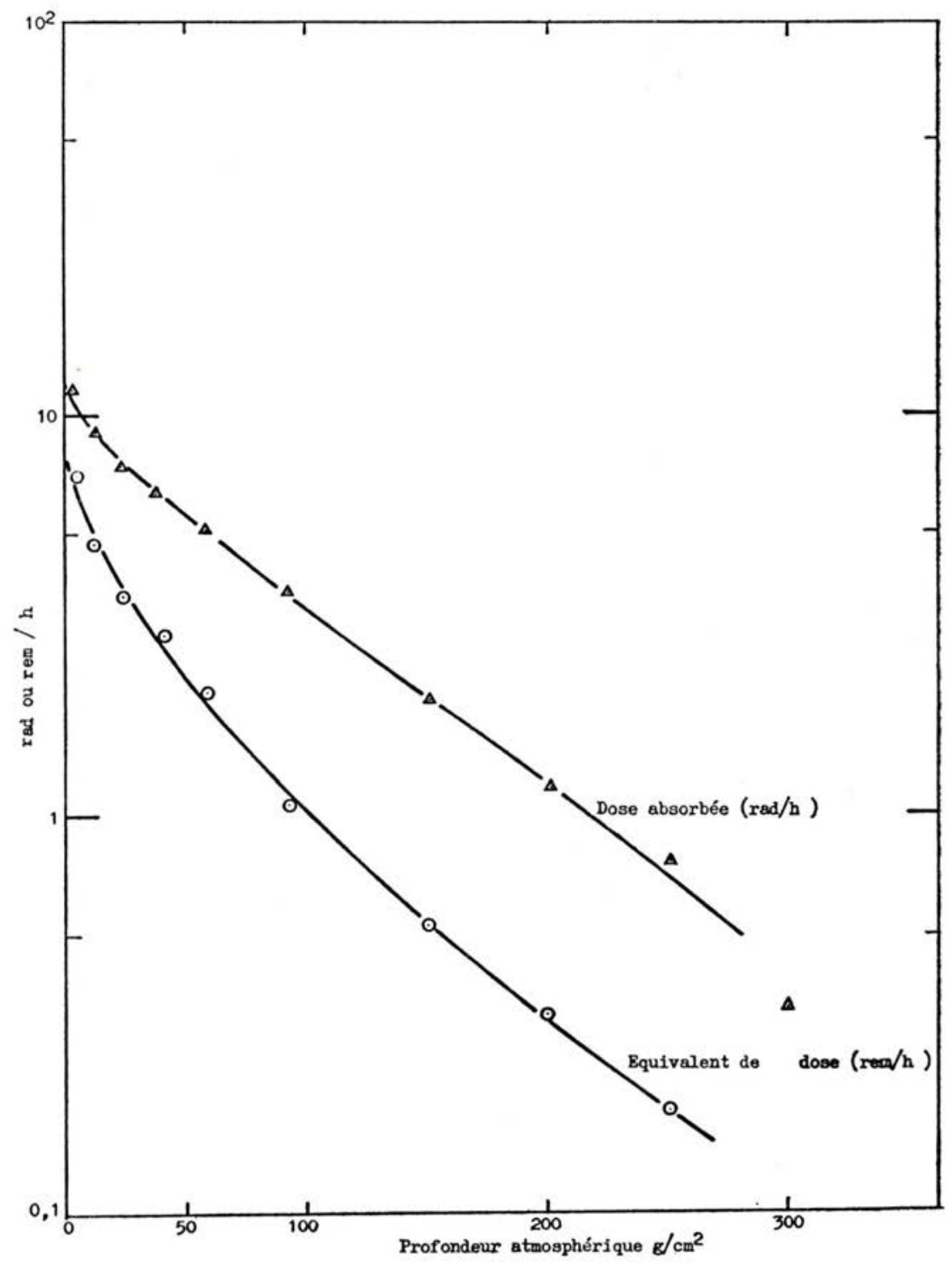

FIG. 15. - Variation du débit d'équivalent de dose maximal avec la profondeur atmosphérique pour l'événement solaire à protons du 23 février 1956, d'après ARMSTRONG et MORAN [3].

reçues aux altitudes de croisière des avions subsoniques et supersoniques provient du calcul de la transmission des radiations à travers l'atmosphère (FUller et SimON [21], Foelsche [19]). Comme les protons primaires des 
rayonnements cosmiques solaires ont, par comparaison au RCG, une faible énergie, les neutrons secondaires qu'ils produisent sont beaucoup plus pénétrants que les protons eux-mêmes. Ainsi, le débit de dose provenant des neutrons constitue toujours une partie significative du débit de dose total et aux altitudes dépassant $100 \mathrm{~g} / \mathrm{cm}^{2}$, il en est la majeure partie.

Fuller et Simon [21] ont calculé (tableau II) l'équivalent de débit de dose à l'altitude du Concorde $\left(100 \mathrm{~g} / \mathrm{cm}^{2}\right)$ et à $28000 \mathrm{~m}$ par les événements à protons du $19^{\circ}$ cycle. Toutes les éruptions solaires ne se font pas ressentir aux altitudes parcourues par les avions. Le cycle actuel (le $\left.20^{\mathrm{e}}\right)$ est de faible activité, sauf en août 1972 où se sont produits deux événements à protons de type important. La figure 14 (d'après Fuller) établit le profil de l'équivalent de débit de dose pour deux altitudes $\left(20\right.$ et $\left.100 \mathrm{~g} / \mathrm{cm}^{2}\right)$ en adaptant la méthode de calcul de Fuller et Simon aux données fournies par les satellites.

TABLEAU III

Équivalent de dose (mrem) pour un voyage à diverses altitudes, d'après FulLER [22].

\begin{tabular}{|c|c|c|c|c|}
\hline $\begin{array}{c}\text { Epaisseur de } \\
\text { l'atmosphère } \\
\mathrm{g} / \mathrm{cm}^{2}\end{array}$ & 20 & 100 & 200 & \\
\hline $\begin{array}{c}\text { Latitude } \\
\text { altude } \\
\mathrm{m}\end{array}$ & 28000 & $16-18000$ & $10-12000$ & \\
\hline 75 & 8,8 & 11,5 & 12,8 & Minimum \\
40 & 3,5 & 6,6 & 8,7 & d'activité \\
10 & 1,1 & 2,3 & 3,6 & solaire \\
\hline 75 & 4,6 & 7,1 & 8,9 & Maximum \\
40 & 2,7 & 4,4 & 7,4 & d'activité \\
10 & 0,9 & 2,1 & 3,4 & solaire \\
\hline
\end{tabular}

Armstrong et MorAN [3] ont pu, grâce aux nombreux renseignements scientifiques recueillis au cours de l'événement solaire à protons du 23 février 1956, établir la courbe de la figure 15 . A l'altitude de $100 \mathrm{~g} / \mathrm{cm}^{2}$ l'équivalent de débit de dose est de $1 \mathrm{rem} / \mathrm{h}$.

Le tableau III donne, d'après FULLER [22] les doses reçues pour un voyage de $18000 \mathrm{~km}$ aux altitudes de 20,100 et $200 \mathrm{~g} / \mathrm{cm}^{2}$ sur des routes de latitude élevée, moyenne et équatoriale en supposant que l'avion vole dans ces bandes à 1800,1200 et $600 \mathrm{~km} / \mathrm{h}$.

\section{BIBLIOGRAPHIE}

Il n'était pas possible de réunir dans le cadre de ce travail une bibliographie exhaustive. Nous avons fait un choix des références complétant la synthèse présentée ci-dessus.

VOL. $10-\mathrm{N}^{\circ} 4$ 
[1] Allkofer O. C. Present knowledge on cosmic rays. Aerospace Medical Panel, Luchon, 27 septembre-1 ${ }^{\text {er }}$ octobre 1971. Rapport AGARD-CP 95 Part III.

[2] Programme Spatial français. Rapport au COSPAR, XVII ${ }^{e}$ Assemblée, Sao Paulo, juin 1974, 113-120.

[3] Armstrong T. W., Moran H. S. Calculation of the radiation hazard at supersonic aircraft altitudes produced by an energetic Solar Flare II. Nucl. Sci. Eng., 1970, 42, 41.

[4] Atlan H. Effets biologiques des particules lourdes. Rev. Méd. Aéron., 1966, 5, 45-57.

[5] Atlan H., Deltour G., Pfister A. M., Kaiser R. Problèmes actuels posés par les radiations cosmiques au cours des vols à haute altitude - expérimentation sur les effets biologiques des ions lourds. Bull. Docum. Méd. Air, 1966, 14, 25-28.

[6] Beckman F. N., Bonney C. H., Hunter D. M. Funduscopic alterations in the Rhesus Monkey induced by exposure to heavy ions $\left(0_{8}^{+}\right) 250 \mathrm{MeV}$ Nucleons. Aerospace Med., 1974, 45, 154-160.

[7] Bucker E., Schopper E., Reinholz, Planel H., Soleilhavoup J. P., François H., Portal G. et coll. The Biostack experiments I et II flown on board of Apollo 16 and 17. $X X I^{\mathrm{e}}$ Congrès international de Médecine aéronautique et spatiale, 17-21 septembre 1973, 106-110.

[8] Budinger T. F., Lyman J. T. Y., Tobias C. A. Visual perception of accelerated nitrogen nuclei interacting with the human retina. Nature, 1972, 239, ( $\left.{ }^{\circ} 5369\right), 209-211$.

[9] Buttner K. Radiation effects on man in space. Bull. Amer. Meteor., 1951, 32, 183-185,

[10] Chase H. B. Cutaneous effects of primary cosmic radiations. J. Aviat. Med., 1954. 25, 388-391.

[11] Chase H. B., Straile W. E., Arsenault C. Heavy ion and millibeam irradiation on mammalian tissues. Aerospace Med., 1961, 32, 921-924.

[12] Cowan F. P. Direct measurement of dose equivalent rates in aircraft. Brookhaven National Laboratory Report BNL-17060, 1972.

[13] CurTis H. J. The use of a deuteron microbeam for simulating the biological effect of heavy cosmic ray particles. Rad. Res., 1967, suppl. 7, 250-257.

[14] CuRTIS H. J. The interpretation of microbeam experiments for manned space flight. Rad. Res., 1967, suppl. 7, 258-264.

[15] Davidson P. J. N. Radiation doses rates at supersonic transport altitudes (MOA Grant PD 134/017). Royal Aircraft Etablishment, Farnborough, 1967.

[16] Eugster J. Method for demonstrating the biological effectiveness of cosmic radiation at high altitude. J. Aviat. Med., 1953, 24, 222-226.

[17] Eugster J. Die mutagene Wirkung der kosmischen Strahlung in menschligen Gewebe (Epidermis). Arch. Julius Klaus Stiftung, 1963, 38, 94-110.

[18] Eugster J. Zur Frage der somatischen Spontanmutation. Arch. Julius Klaus Stiftung, 1966, 41, 1-116.

[19] FoelsChe T. Dose response functions in the atmosphere due to incident high energy protons with applications to SPE. NASA report T.N.D-6010, 1970.

[20] Fuller E. W., Clarke N. T. The radiological dose to the SST Concorde. United Kingdom Atomic energy authority report AWRE 064/68, 1968.

[21] Fuller E. W., Simon P. Frequency of solar proton events at Concorde altitudes (communication personnelle).

[22] Fuller E. W. Doses dues aux rayonnements cosmiques aux altitudes inférieures à $25 \mathrm{~km}$. Rapport final du groupe de travail “ Biophysical Problems » de l'Aerospace Medical Panel AGARD (OTAN) (à paraitre). 
[23] GyURdian A. A. Sur l'action biologique des radiations cosmiques. Satellittes artificiels de la Terre, U.R.S.S., 1962, 12, 77-104.

[24] HaYmakeR W. et coll. Brain study in balloon monkeys exposed to cosmic rays. Aerospace Med., 1970, 41, 989-1002.

[25] HAYMAKer W. et coll. 26 Biocore Experiment 1974. NASA-APOLLO 17 Preliminary science Report (26.1-14).

[26] Langham W. H. Radiobiological factors in manned space flight. Report of the space radiation study panel of the Life Sciences Committee, Space Science Board. National Academy of Sciences, National Research Council, Washington DC, 1967.

[27] McNulty P. J., Pease V. P., Pinsky L. S., Bond V. P., Schimmerling W., VosBuRgh K. G. Visual sensations induced by relativistic nitrogen nuclei. Science, 1972, 178, 160-162.

[28] NeHER H. V. Cosmics rays at high latitudes and altitudes covering four solar maximum. J. Geophys. Res., 1971, 76, 1637.

[29] O'Brien K., McLaughin J. E. Calculation of dose and dose equivalent rates to man in the atmosphere from galactic cosmic rays. USAEC report HASL-228, 1970.

[30] Pfister A. M., Deltour G., Atlan H., Kaiser R. Méthode expérimentale pour l'étude des effets biologiques des ions lourds du rayonnement cosmique primaire sur les bactéries. $X V^{\mathrm{e}}$ Congrès international de Médecine aéronautique et cosmonautique, Prague, octobre 1966.

[31] Pfister A. M., Delahaye R. P. Dangers présentés par les ions lourds du rayonnement cosmique pour les TSS. Rev. Méd. Aéron., 1967, 6, 33-34.

[32] Pipkin S. B., Sullivan W. N. A search for genetic change in Drosophila melanogaster exposed to cosmic radiation at extreme altitude. Aerospace Med., 1959, 30, 585-598.

[33] Planel H., Soleilhavoup J.-P., Tixador R., Giess M. C., Croute F. Mise en évidence d'un effet biologique de stimulation des radiations ionisantes naturelles. Aerospace Medical Panel, Luchon, 27 septembre-1 $^{\text {er }}$ octobre 1971. Rapport AGARD-CP 95, C 5-1 à C 5-11.

[34] SCHAEFfer H. J. Radiation measurement at supersonic transport altitude with balloonborne nuclear emulsion. Rapport NAMI-1068, 1969.

[35] SHEN S. P. Space radiation and the supersonic transport altitudes. Final report on M OA Grant PD 134/017. Royal Aircraft Etablishment, Farnborough, 1967.

[36] Simons D. G. Biological effects of primary cosmic radiation. Proc. VII International Astronautical Congress, 1956, 381-400.

[37] Simons D. G. Biological hazards of space radiations. Med. Phys., vol. III, The Year Books, 1960.

[38] Simons D. G., Hewitr J. E. Symposium on aerospace radiobiology VII - Review of biological effects of galactic cosmic radiation. Aerospace Med., 1961, 32, 932-941.

[39] TABusse E. M. Contribution à l'étude des problèmes radiobiologiques posés par les rayonnements extraterrestres. Thèse Médecine, Paris, 1964.

[40] Tobias C. A., Budinger T. F., Leith J. T., Mamoon A. M., Chapman P. Visual phenomenon induced by cosmic rays and accelerated particles. Aerospace Medical Panel, Luchon, 27 septembre-1 $^{\text {er }}$ octobre 1971. Rapport AGARD-CP 95, C 6-1 à C 6-11.

[41] Delahaye R. P., Pfister A. Études biologiques des rayonnements cosmiques. Rev. Méd. Aéron., 1974, 13, 276-283. 